\title{
Approaches to closed-loop deep brain stimulation for movement disorders
}

\author{
Chao-Hung Kuo, MD,1-3 Gabrielle A. White-Dzuro, MD,1 and Andrew L. Ko, MD1,4 \\ ${ }^{1}$ Neurological Surgery, University of Washington, Seattle, Washington; ${ }^{2}$ Department of Neurosurgery, Neurological Institute, \\ Taipei Veterans General Hospital, Taipei, Taiwan; ${ }^{3}$ School of Medicine, National Yang-Ming University, Taipei, Taiwan; and ${ }^{4}$ NSF \\ Engineering Research Center for Sensorimotor Neural Engineering, Seattle, Washington
}

\begin{abstract}
OBJECTIVE Deep brain stimulation (DBS) is a safe and effective therapy for movement disorders, such as Parkinson's disease (PD), essential tremor (ET), and dystonia. There is considerable interest in developing "closed-loop" DBS devices capable of modulating stimulation in response to sensor feedback. In this paper, the authors review related literature and present selected approaches to signal sources and approaches to feedback being considered for deployment in closed-loop systems.
\end{abstract}

METHODS A literature search using the keywords "closed-loop DBS" and "adaptive DBS" was performed in the PubMed database. The search was conducted for all articles published up until March 2018. An in-depth review was not performed for publications not written in the English language, nonhuman studies, or topics other than Parkinson's disease or essential tremor, specifically epilepsy and psychiatric conditions.

RESULTS The search returned 256 articles. A total of 71 articles were primary studies in humans, of which 50 focused on treatment of movement disorders. These articles were reviewed with the aim of providing an overview of the features of closed-loop systems, with particular attention paid to signal sources and biomarkers, general approaches to feedback control, and clinical data when available.

CONCLUSIONS Closed-loop DBS seeks to employ biomarkers, derived from sensors such as electromyography, electrocorticography, and local field potentials, to provide real-time, patient-responsive therapy for movement disorders. Most studies appear to focus on the treatment of Parkinson's disease. Several approaches hold promise, but additional studies are required to determine which approaches are feasible, efficacious, and efficient.

https://thejns.org/doi/abs/10.3171/2018.5.FOCUS18173

KEYWORDS closed-Ioop DBS; adaptive DBS; kinematic sensors; electrocorticography; local field potentials; deep brain stimulation

$\mathrm{D}$ EEP brain stimulation (DBS) is a safe and effective therapy for movement disorders, such as Parkinson's disease (PD), essential tremor (ET), and dystonia. ${ }^{2,715}$ The devices have been in clinical use for decades, providing invariant stimulation at a fixed spatial distribution (electrode configuration), amplitude, frequency, and pulse width. This "open-loop" therapy relies on the determination of effective stimulation parameters by a clinician. As our understanding of the mechanisms underlying this therapy and movement disorders in general expands, the shortcomings of this system are increasingly evident.

Although DBS provides effective treatment of the motor symptoms of diseases such as PD, side effects of therapy can include cognitive impairment and changes in speech, gait, and balance. ${ }^{21}$ While most patients deem such side effects tolerable,$^{20}$ the current approach likely does not restore basal ganglia function to the greatest extent possible, given its static approach to therapy within an inherently dynamic system. Moreover, the use of constant stimulation provides stimulation in excess of what is clinically warranted. With the most-commonly implanted, nonrechargeable DBS systems, this excess power usage shortens battery life and exposes patients to the risks of surgical replacement of the implantable pulse generator.

ABBREVIATIONS AP = action potential; DBS = deep brain stimulation; ECoG = electrocorticography; EEG = electroencephalography; EMG = electromyography; ET = essential tremor; IMU = inertial monitoring unit; LDF = linear delayed feedback; LFP = local field potential; PD = Parkinson's disease; sEMG = surface EMG; STN = subthalamic nucleus; VIM = ventral intermediate nucleus.

SUBMITTED April 2, 2018. ACCEPTED May 11, 2018.

INCLUDE WHEN CITING DOI: 10.3171/2018.5.FOCUS18173. 
While cardiac pacemaker devices capable of sensing and responding to patient physiology have been in clinical use for over 50 years, ${ }^{41}$ similar efforts to develop a "closedloop" DBS device have been delayed; this is likely due to the complexity of brain signals and uncertainty surrounding the clinical significance of recordable brain activity. These roadblocks are increasingly surmountable, with advances in technology, development of applicable algorithms, and a greater understanding of neurophysiology.

Closed-loop DBS represents a subset of bidirectional brain computer interfaces; a comprehensive review of such systems is beyond the scope of this paper, which will focus on relatively well-established approaches to the treatment of movement disorders. The goal is to provide an overview of the features of a closed-loop system, with attention paid to signal sources and biomarkers, general approaches to feedback control, and clinical data when available.

\section{Methods}

A literature search using the keywords "closed-loop DBS" and "adaptive DBS" was performed using the PubMed database. The search was conducted for all articles published up until March 2018.

The keywords "closed-loop DBS" resulted in 156 articles, while "adaptive DBS" returned 100 articles. The procedure for narrowing these results for in-depth review is presented in Fig. 1.

\section{General Description of a "Closed-Loop" Approach}

A general control system diagram for a putative closedloop DBS system is presented in Fig. 2. First, the signal is extracted from sensor data and used to predict the patient's current state. This signal can include data derived from an external sensor (e.g., electroencephalography [EEG] or inertial measurement unit [IMU]), or implanted sensor (e.g., electrocorticography [ECoG], local field potentials [LFPs], or action potentials [APs]). The system must identify useful features, including, for example, the desynchronization of neural oscillations. Algorithms employing models of neurochemical or electrophysiological dynamics allow generation of an estimated clinical state, which in turn drives selection and optimization of stimulation parameters in order to achieve desired features in the reference signal. The controller examines the reference signal and the desired features, calculates error, and outputs a control signal that is passed to the actuator, which applies the predicted beneficial stimulation parameters. This ultimately influences the signals detected by the sensor, serving as a surrogate for the benefit of DBS, bringing the detected reference signal and desired features closer together (Fig. 2).

\section{Results}

\section{Approaches to Biomarkers: Available Signals and Features}

Various biological signals, including kinematic data (accelerometer) and those obtained using electromyography (EMG), EEG, ECoG, LFPs, and APs, have been considered as biomarkers for closed-loop DBS (Table 1). Each varies with respect to invasiveness, resolution, signal content, and clinical relevance. For example, EEG electrodes are noninvasive but measure voltage changes from a large

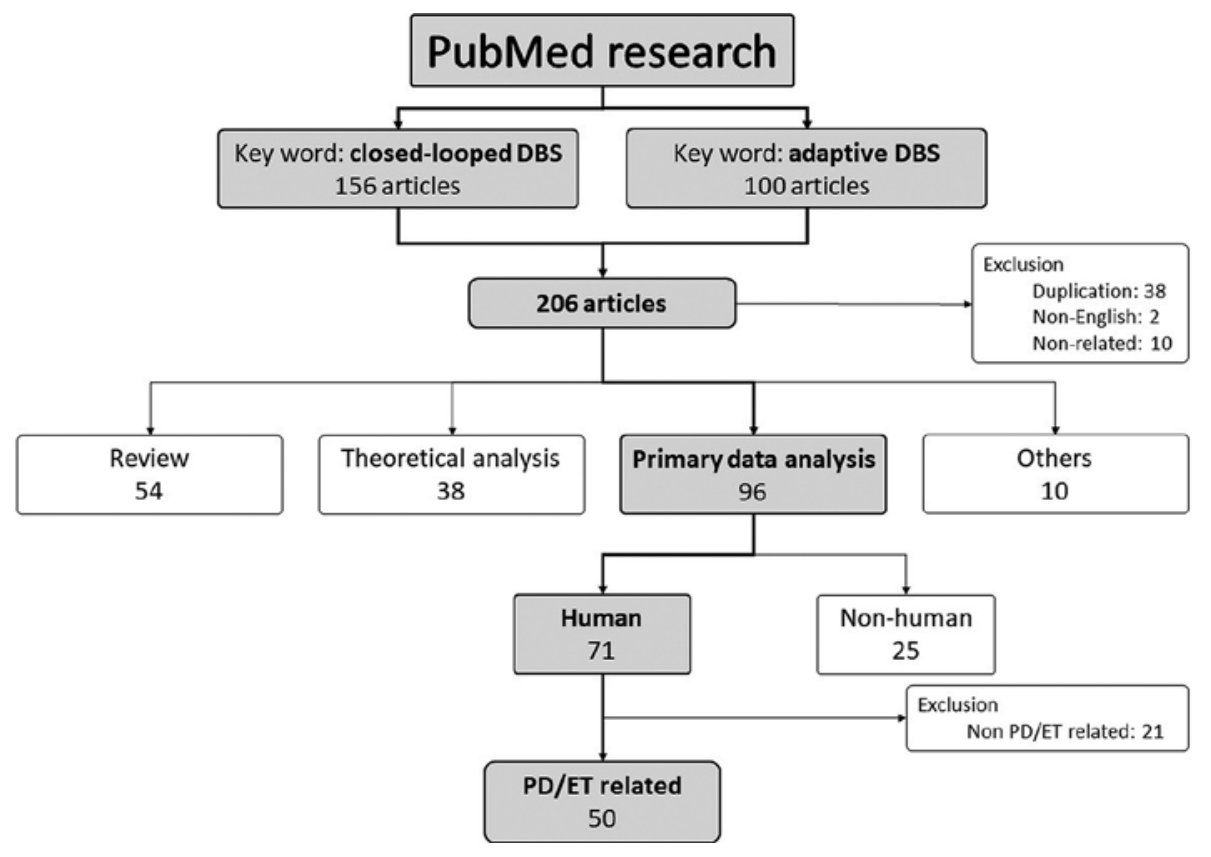

FIG. 1. A flow diagram illustrating the types of papers included and excluded for reviewing in this article. The search terms "closedloop DBS" and "adaptive DBS" returned 156 and 100 articles, respectively. Duplicate articles, non-English language articles, and articles clearly not related to DBS were excluded. Primary data analyses were divided into human and nonhuman studies, resulting in 71 articles. Those related to treatment of movement disorders (50 total) were included for in-depth review. 


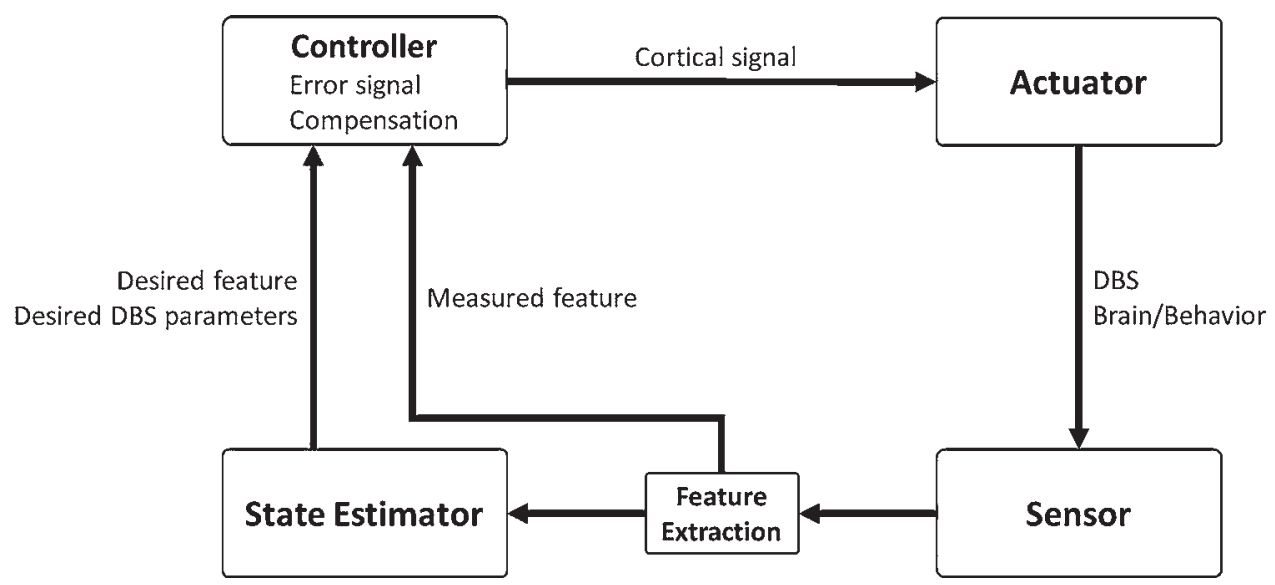

FIG. 2. General diagram for conceptualizing the closed-loop DBS system. Data gathered by a sensor is converted into a measured feature. This reference signal is passed to a state estimator, which generates a desired feature and desired DBS parameters. The controller compares the reference signal and desired signal, generates an error signal, and compensates to generate a control signal. This is passed on to the actuator, which influences stimulation at the basal ganglia. The effects on brain and behavior are detected by the sensor, which provides closed-loop feedback into the system.

volume, with little usable signal at high frequencies. ${ }^{6} \mathrm{~A}$ microelectrode capable of measuring APs will have a small contact area, allowing for very high spatial and frequency resolution. ${ }^{38}$ On the other hand, AP recordings from small groups of neurons can change from day to day, which may limit their application for long-term stimulation. ${ }^{45}$ Signals such as accelerometer data are noninvasive, but such kinesthetically rich information can be noisy and

TABLE 1. Examples of sensors used for closed-loop DBS

\begin{tabular}{|c|c|c|c|c|}
\hline Type of Biomarker & Signal Source & Characteristics & Pt Pop & Authors \& Year \\
\hline \multirow[t]{4}{*}{ Kinematic data } & \multirow{4}{*}{$\begin{array}{l}\text { Accelerometer or } \\
\text { gyroscope }\end{array}$} & \multirow{4}{*}{$\begin{array}{l}\text { Pros: noninvasive, unchanged surgical procedure. } \\
\text { Cons: wireless transmission shortens life of battery. }\end{array}$} & PD & Shukla et al., 2012 \\
\hline & & & $\mathrm{PD}$ & Malekmohammadi et al., 2016 \\
\hline & & & ET & Herron et al., $2017^{22}$ \\
\hline & & & DT/ET & Cagnan et al., 2017 \\
\hline \multirow[t]{5}{*}{ EMG } & \multirow[t]{5}{*}{ Forearm muscle } & \multirow{5}{*}{$\begin{array}{l}\text { Pros: noninvasive; unchanged surgical procedure. } \\
\text { Cons: signals easily affected by noise. }\end{array}$} & ET & Graupe et al., 2010 \\
\hline & & & PD & Shukla et al., 2012 \\
\hline & & & ET/PD & Basu et al., 2013 \\
\hline & & & ET & Yamamoto et al., 2013 \\
\hline & & & ET & Herron et al., $2017^{22}$ \\
\hline \multirow[t]{6}{*}{ ECoG } & \multirow{6}{*}{$\begin{array}{l}\text { Primary sensorimotor } \\
\quad \text { cortex }\end{array}$} & \multirow{6}{*}{$\begin{array}{l}\text { Pros: well-developed recording device \& technique. } \\
\text { Cons: invasive, extra surgical procedure for electrodes } \\
\text { implanted. }\end{array}$} & ET & Air et al., 2012 \\
\hline & & & PD & de Hemptinne et al., 2013 \\
\hline & & & ET/PD & Rowland et al., 2015 \\
\hline & & & ET/PD & Kondylis et al., 2016 \\
\hline & & & PD & Swann et al., 2016 \\
\hline & & & ET & Herron et al., $2017^{22}$ \\
\hline \multirow[t]{7}{*}{ LFP } & \multirow[t]{7}{*}{ Basal ganglion } & \multirow{7}{*}{$\begin{array}{l}\text { Pros: unchanged surgical procedure for implantation. } \\
\text { Cons: signal recording can be affected by lesion effect; } \\
\text { simultaneous sensing \& stimulation can be difficult. }\end{array}$} & PD & Priori et al., 2004 \\
\hline & & & PD & Brown \& Williams, 2005 \\
\hline & & & PD & Wingeier et al., 2006 \\
\hline & & & ET & Air et al., 2012 \\
\hline & & & PD & Niketeghad et al., 2014 \\
\hline & & & $\mathrm{D} / \mathrm{PD}$ & Mamun et al., 2015 \\
\hline & & & PD & Quinn et al., 2015 \\
\hline \multirow{2}{*}{$\begin{array}{l}\text { Neurochemical } \\
\text { dynamics }\end{array}$} & \multirow[t]{2}{*}{ Neurotransmitter level } & \multirow{2}{*}{$\begin{array}{l}\text { Pros: real-time monitoring. } \\
\text { Cons: no mature human study model. }\end{array}$} & PD & Kishida et al., 2011 \\
\hline & & & PD & Lee et al., $2017^{\star}$ \\
\hline
\end{tabular}

$\mathrm{D}=$ dystonia; $\mathrm{DT}$ = dystonic tremor; pt pop = patient population

* Nonhuman animal model-based study. 
requires telemetry between an external device and an implanted neurostimulator, driving up energy costs.

\section{Feature Extraction and Classification}

Feature extraction transforms time-series data for subsequent processing and potentially improves data processing efficiency. Signals can be examined in the time, frequency, or time-frequency domain. For example, the oscillatory activity of neuronal ensembles can be characterized by techniques to estimate spectral power in the frequency domain, such as short-time Fourier transform or wavelet transformations, and changes within particular frequency bands have been correlated to behavior in well-defined regions of the cortex and subcortical structures. We will discuss a subset of these techniques in the context of particular studies. Likewise, there are many approaches to the identification and segregation of patterns used to generate state estimations for closed-loop control. Suffice it to say that some of these algorithms, such as linear discriminant analysis, may be embedded within DBS devices, such as the Activa PC+S, to classify signals and adapt to dynamic patient requirements.

\section{Kinematic Data}

Data from accelerometers or IMUs can be used to detect symptoms such as tremor. A feedback loop based on the presence or absence of tremor or the onset of movement, can be used to control an adaptive DBS device. One example of both approaches can be seen in a case report by Herron et al., ${ }^{22}$ which illustrates several advantages and disadvantages of this approach.

IMU data were collected using a smartwatch worn on the affected limb. These data were processed and fed into a control algorithm adjusting stimulation according to the magnitude of the tremor band power $(4-8 \mathrm{~Hz})$ on the 3 gyroscope channels. This tremor-modulated approach reduced the average tremor band amplitude by about $59 \%$ when compared to open-loop DBS, but it used only $16 \%$ of baseline stimulation, representing a $6.5 \%$ gain in efficiency for every $1 \%$ increase in tremor (Fig. 3). ${ }^{22}$

A similar, tremor-modulated approach has been used in PD patients. ${ }^{35}$ Stimulation amplitude was modulated as a proportion of detected tremor using an IMU, resulting in a $37 \%$ reduction in average tremor amplitude with an energy savings of more than $75 \%$. An important difference in considering these results is the stochastic, unpredictable nature of resting tremor in PD patients versus the predictable, movement-associated tremor in ET.

In an alternative approach, Cagnan et al. extracted the dominant phase of tremor in ET patients. Low-frequency (approximately $4 \mathrm{~Hz}$ ), phase-locked stimulation was provided at randomized phases relative to the tremor phase, and the most effective phase-offset was determined empirically. Phase-locked DBS was then delivered during tremor-provoking posture holding. This approach achieved up to $87 \%$ symptom suppression. ${ }^{11}$ Power savings in this system are achieved using a lower stimulation frequency. Another potential benefit of this phase-locked approach is mitigation of stimulation-related side effects; in principle, functional networks characterized by oscillatory networks are not affected by stimulation if they are not entrained to the same phase as the patient's tremor.
Data from kinematic sensors, such as accelerometers, have revealed quantitatively significant differences between patients with PD in "on" and "off" medication states, and also between PD patients and healthy individuals. ${ }^{8}$ Preliminary data on the use of smartphones to gather similar kinematic data show that symptom severity can be predicted for modalities, such as gait,${ }^{16}$ bradykinesia, ${ }^{29}$ and dyskinesias..$^{53}$ Data of this type could potentially be used to provide a viable reference signal for adaptive DBS systems, using individual patient models of the disease state to modulate delivery of DBS.

\section{Electromyography}

Surface EMG (sEMG) from symptomatic extremities can provide useful information as a biomarker in closedloop DBS. ${ }^{54}$ Graupe et al. performed a trial in which a patient undergoing DBS for ET underwent test stimulation with simultaneous sEMG monitoring. The signals from $\mathrm{SEMG}$ were filtered by 4 frequency bands $(1-2,2-4$, $4-8$, and $8-16 \mathrm{~Hz}$ ) and analyzed by discrete wavelet transforms, which revealed that the activated power of bandpass sEMG signals $(4-8 \mathrm{~Hz})$ could be used to predict the onset of tremor before tremor appeared. ${ }^{19}$

Several case series have implemented the use of sEMG as a sensor for closed-loop DBS.22,62 Yamamoto et al. used power in a $3-\mathrm{Hz}$ tremor band to trigger stimulation. ${ }^{62}$ Another study examining ET and PD patients employed wavelet entropy for measuring the similarity and difference between different segments of the signal. The power band in the 8- to $16-\mathrm{Hz}$ range showed high predictive value for detecting tremor, achieving $100 \%$ sensitivity and $85.7 \%$ accuracy for ET and $80.2 \%$ accuracy for PD trials. ${ }^{5}$ These symptom-modulated approaches rely on the presence of tremor to initiate stimulation.

Another approach is to initiate stimulation in a movement-modulated fashion, providing DBS throughout any detected movement. Using surface EMG, Herron et al. ${ }^{22}$ showed that this approach provided $92 \%$ of the tremor control generated by open-loop DBS with 53\% of the power usage, resulting in a 2:1 gain in efficiency. This approach was compared with a tremor-modulated, IMU-based, closedloop model, detailed in the previous section. Both methods were $100 \%$ accurate in detecting either movement or rest conditions, on a per-epoch basis, showing that kinematic control for closed-loop DBS can be very accurate. These data illustrate an interesting trade-off inherent in approaches that detect the presence or absence of a symptom: in order for the IMU-based, tremor-modulated system to provide stimulation, some tremor must be present, entailing poorer tremor control. Movement-modulated stimulation, on the other hand, provided better tremor control at the cost of a higher rate of power consumption. However, noise in the EMG signal can be caused by small movements of the electrodes, making it potentially difficult to extract a reliable signal during real-world use. ${ }^{3}$ Moreover, reliable sEMG markers for cardinal symptoms of PD, such as rigidity and bradykinesia, have not yet been developed.

\section{Electrocorticography}

Electrocorticography has been widely studied in patients with epilepsy $y^{4,55}$ and is currently used as a biomarker 


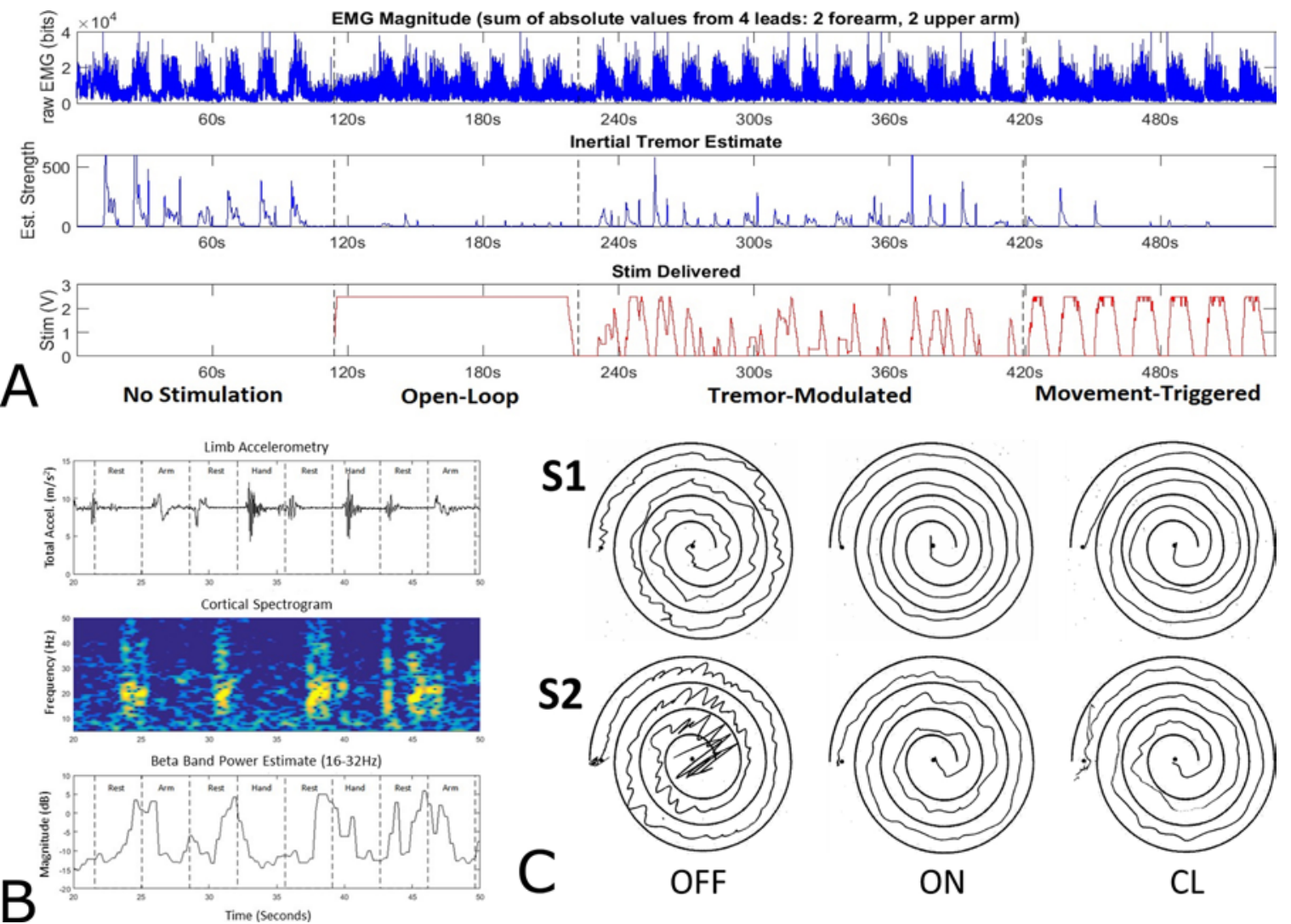

FIG. 3. Closed-loop DBS for essential tremor. A: Kinematic control of a closed-loop DBS system for essential tremor. Data are shown shown during the prompted tremor-provoking movement task under no stimulation, open-loop stimulation, tremormodulated stimulation (IMU tremor-band), and movement-modulated (EMG amplitude threshold) DBS. Upper: EMG recording from right arm during prompted movement task. Center: Tremor magnitude estimate from the wrist-worn IMU. Lower: Magnitude of DBS provided during no stimulation, open-loop stimulation, tremor-modulated closed-loop stimulation, and movement-triggered closed-loop stimulation. Note the excellent tremor control during EMG-modulated closed-loop DBS. B: IMU readings (upper) with concurrent ECoG spectrogram (center), and beta-band-limited power (lower) during prompted arm movement task. The beta-band power was thresholded and used for closed-loop control of DBS for ET. C: Example spiral drawing under no stimulation, open-loop DBS, and cortically controlled closed-loop (CL) DBS in 2 patients (S1 and S2). Open-loop and closed-loop drawings are nearly identical. Accel. = acceleration; Est. = estimated; stim. = stimulation.

in a brain-responsive neurostimulator (RNS system, Neuropace).$^{18}$ While widely considered a disease affecting the basal ganglia, there is evidence that information regarding the disease state can be extracted from cortical signals using ECoG.

PD patients show larger beta desynchronization in early motor preparation and higher gamma power during rest and movement when compared with ET patients..$^{52}$ Other studies have shown excess in PD patients; beta-band phase (13-30 Hz) modulation of gamma-band amplitude (50$200 \mathrm{~Hz}$ ) was observed in the primary motor cortex (M1) of patients at rest, but this coupling is significantly stronger in PD patients. ${ }^{13,27}$ Interestingly, phase-amplitude coupling within M1 decreased in PD patients during effective DBS, both at rest and during movement. These studies provide evidence that cortical signals are a potential biomarker for successful treatment of symptoms with DBS.

Recent studies using the Activa $\mathrm{PC}+\mathrm{S}$ system offer the ability to perform long-term, multisite recording in DBS patients. In one study, the electrodes for DBS were implanted at the subthalamic nucleus (STN), with ipsilateral cortical electrodes overlying the sensorimotor cortex. The presence of dyskinesias was highly correlated with narrowband gamma oscillations in the motor cortex between 60 and $90 \mathrm{~Hz}$, independent of voluntary movements..$^{56}$ During therapeutic DBS, when dyskinesias are present, the narrowband gamma oscillations shift in frequency to one-half the stimulation frequency. This finding suggests a viable cortical biomarker for closed-loop DBS with respect to the treatment of dyskinesias.

An alternative to using ECoG-derived LFPs as markers for disease state is their use to detect behavior associated with the need for DBS. In an ET study using the Medtronic $\mathrm{PC}+\mathrm{S}$ system, a cortical strip was placed over the hand sensorimotor cortex in addition to the DBS electrode in the thalamus of the ventral intermediate nucleus (VIM). Movement-related beta-band desynchronization in the cortical signal was used to drive stimulation at the VIM; this system provides closed-loop stimulation based on the premise that therapy for ET is required only during intentional movement. Evaluation of the accuracy of the controller showed that stimulation was on for $100 \%$ 
of prompted movement tasks and was on throughout tasks such as spiral drawing or water pouring. ${ }^{23}$

This demand-driven paradigm for ET did reveal challenges of an "on-off" approach to closed-loop DBS: individual sensitivity to the rate of amplitude changes must be taken into consideration. Use of a bipolar electrode configuration mitigates paresthesias during initiation of stimulation. More complex controller algorithms are also being developed to alleviate the need to turn a system from a fully "off" to a therapeutic stimulation level. In general, limiting the slew rate can avoid paresthesias, and, while this results in some tremor at movement initiation, it is quickly suppressed. Interestingly, this initial presence of tremor may be difficult to detect for both the patient and expert clinicians; the difference in tremor control between using constant DBS and closed-loop DBS employing this demand-driven strategy was indistinguishable to blinded clinical raters (manuscript in submission).

\section{Subcortical Local Field Potential}

Local field potentials reflect neural processes occurring in the extracellular space around a recording electrode. ${ }^{24}$ Beta-band power within the STN is the most explored use of LFPs as a biomarker for disease state in PD. The link between beta-band power in the STN and motor state in PD has been demonstrated in a large cohort of patients, with correlation between $8-35 \mathrm{~Hz}$ band-limited power in the STN and UPDRS (United Parkinson's Disease Rating Scale)-III scores seen in all of a series of 63 patients. ${ }^{42}$ This finding has been corroborated in patients implanted with the Activa $\mathrm{PC}+\mathrm{S}$ system, where peaks in the 13- to $35-\mathrm{Hz}$ range were reliably seen, suppressed by levodopa, and correlated with parkinsonian motor impairment on the individual level across time points and dopaminergic states. $^{43}$

The suitability of using beta-band power thresholds to trigger STN stimulation has been demonstrated in humans in the acute setting. ${ }^{32,33}$ In a series of studies, LFPs were recorded from the STN, bandpass-filtered between 3 and $37 \mathrm{~Hz}$, and converted to beta amplitude by rectifying and smoothing. Monopolar stimulation was triggered when beta power crossed a user-defined threshold and terminated while beta power dropped below threshold. The results were superior to those of traditional open-loop stimulation on blinded assessment. For unilateral therapy, a $50 \%$ reduction in UPDRS-III scores was seen during closed-loop stimulation compared with $30 \%$ improvement during open-loop stimulation, with a reduction in stimulation time of $44 \% .{ }^{33}$ Importantly, similar results were not seen with random, intermittent stimulation, suggesting that intermittency alone was not responsible for the additional benefit. Similar results were noted during bilateral therapy. ${ }^{32}$ These studies employed a straightforward control algorithm, with voltage adjustments designed to elicit clinical benefit without paresthesias, at a threshold for stimulation heuristically determined to provide about $50 \%$ time-on-stimulation. Interestingly, even with this simple algorithm, the effect of levodopa administration was detectable as significantly decreased time-on-stimulation based on beta-band power, without a deterioration in motor state, showing that the beta-band signal used for closed-loop control accurately reflected the clinical need for stimulation in the face of medication administration. ${ }^{32}$

Notably, follow-up studies have shown that a significant improvement in speech intelligibility was seen during closed-loop DBS when compared with conventional therapy. ${ }^{34}$ These impressive results have been described in patients with externalized DBS leads, with the attendant confounds of DBS in the acute setting. Longer-term studies are in progress, and the ability of closed-loop DBS to provide similar or superior motor benefit, an improved side-effect profile, with a time-on-stimulation on the order of $45 \%$ when compared to constant stimulation, is an exciting prospect.

\section{Neurochemical Dynamics}

Dopamine has an important role in motor and cognitive function and has long been implicated in the pathophysiology of PD. ${ }^{14,39,58}$ It stands to reason that dopamine dynamics may be a potential biomarker for a closed-loop system. The development of fast-scan cyclic voltammetry at carbon microelectrodes provides a method for real-time in vivo measurement of neurochemical changes in dynamic brain processes; refinement of these systems has made it possible to measure response to pharmacological, cognitive, behavioral, and neuromodulatory interventions, such as DBS. ${ }^{31}$

In humans, this methodology has been applied to detect rapid (subsecond) measurements of dopamine release in a PD patient during decision-making tasks. ${ }^{26}$ The relationship between the dynamics of dopamine release and DBS at the STN has not been elucidated in humans; however, detectable dopamine increases at "therapeutic" charge densities applied during DBS have been observed in the porcine STN. ${ }^{30}$ Such a real-time monitor of dopamine levels provides a potential method for closed-loop control of a DBS system.

Similar methodology has been applied in patients undergoing DBS for ET. In 8 patients, localized release of adenosine was noted during placement of a DBS electrode at the VIM, with some patients showing a voltagedependent response to DBS at the same site..$^{2}$ This study, however, did not correlate adenosine release with tremor reduction.

\section{Approaches to Feedback and DBS Parameter Modulation}

Open-loop DBS requires neurologists to adjust stimulation parameters and evaluate results in an outpatient clinical setting. Selectivity and efficiency are important in determining optimal stimulation parameters; the goal is to use the minimum amount of stimulation to achieve clinical effect by activating the targeted neural elements without nontarget effects.

Current clinical DBS systems deliver a constant train of pulses, with adjustments possible in spatial application across electrode configurations and variation of amplitude, pulse-width, and frequency of the stimulation train. In principle, any of these parameters may be modified using a closed-loop approach (Table 2).

\section{Amplitude Response}

Potentially, the most straightforward approach involves 
TABLE 2. Examples of approaches to feedback

\begin{tabular}{|c|c|c|c|c|}
\hline Feedback Type & Biomarker & Pt Pop & Description & Authors \& Year \\
\hline \multirow{4}{*}{$\begin{array}{l}\text { Amplitude } \\
\text { response }\end{array}$} & LFP & PD & Beta-band amplitude threshold triggered STN DBS & Little et al., 2013 \\
\hline & EMG & ET & Stimulation on/off based on 3-Hz power threshold in sEMG & Yamamoto et al., 2013 \\
\hline & LFP & PD & Beta-band amplitude threshold triggered STN DBS & Little et al., $2016^{34}$ \\
\hline & $\begin{array}{r}\text { ECoG, EMG, } \\
\text { gyroscope }\end{array}$ & ET & $\begin{array}{l}\text { EMG: movement threshold; IMU: amplitude threshold in } 4-8 \mathrm{~Hz} \text { "tremor } \\
\text { band"; ECoG: cortical beta-desynchronization triggered stimulation }\end{array}$ & Herron et al., $2017^{22}$ \\
\hline \multirow{6}{*}{ Model-based } & Theoretical & PD & Amplitude of phase-shifted "passive oscillator" & Tukhlina et al., $2007^{*}$ \\
\hline & Accelerometer & ET & Phase-shifted stimulation at dominant tremor frequency & Cagnan et al., 2013 \\
\hline & LFP, EMG & PD & $\begin{array}{l}\text { Low-frequency irregular patterns of stimulation \& low-frequency periodic } \\
\text { stimulation interrupted by bursts of pulses exerted the strongest desyn- } \\
\text { chronizing effect }\end{array}$ & Karamintziou et al., 2016 \\
\hline & Accelerometer & DT/ET & Low-frequency stimulation phase-locked to tremor frequency & Cagnan et al., 2017 \\
\hline & Theoretical & PD & $\begin{array}{l}\text { Pulsatile delayed feedback stimulation used to desynchronize a network btwn } \\
\text { STN \& GPe }\end{array}$ & Popovych et al., $2017^{46,47 *}$ \\
\hline & Theoretical & PD & Pulsatile multisite LDF modulating the pulse amplitude of high-frequency DBS & Popovych et al., 2018* \\
\hline
\end{tabular}

$\mathrm{GPe}=$ external globus pallidus .

* Based on a simulated neuronal network mimicking a parkinsonian condition.

an amplitude response: stimulation parameters are determined using traditional programming, and closed-loop control merely modulates stimulation amplitude in response to an estimated clinical state.

Most of the more mature approaches to closed-loop DBS take this approach, employing either an event-dependent, on/off control, or continuous-time control where stimulation amplitude varies proportionately to the amplitude of the signal. As described above, efficacy of this approach to feedback has been employed with kinematic markers in $\mathrm{PD}^{35}$ and ET, ${ }^{22}$ electrocorticography in ET, ${ }^{23}$ and STN LFPs in PD. ${ }^{32,33}$

\section{Model-Based Approaches}

The cardinal symptoms of PD can be conceived of as the consequence of pathological synchronization of neural oscillations in a widespread brain network. Intraoperative recordings demonstrate that while pathologic beta-band oscillations in STN LFPs may be generated locally, ${ }^{59}$ this excessive synchronization can be detected outside the basal ganglia. ${ }^{9}$ Karamintziou et al. illustrated a data-driven computational model by using neuronal synchronization dynamics within and outside the STN. Stimulation patterns were designed with various stimulation frequencies $(80 \mathrm{~Hz}$ and $130 \mathrm{~Hz})$ and temporal regularity. Low-frequency, irregular patterns of stimulation and low-frequency periodic stimulation interrupted by bursts of pulses exerted the strongest desynchronizing effect on neuronal activity. Thus, detecting and disrupting this network of oscillatory neurons is potentially another strategy for feedback control. ${ }^{25}$

Popovych et al. presented a delayed feedback stimulation method, which combined high-frequency DBS stimulation and pulsatile delayed feedback to desynchronize abnormal neuronal activity. ${ }^{46,47}$ Such methods include pulsatile linear delayed feedback (LDF) or pulsatile nonlinear delayed feedback as methods to use to counteract abnormal neuronal synchronization present in PD. Further studies have employed pulsatile multisite LDF by using signal not only from the STN but also external globus pallidus neurons. However, the efficacy of the pulsatile multisite LDF in inducing desynchronization was much lower than that of the pulsatile $\mathrm{LDF}^{48}$

The concept of vanishing-stimulation control ${ }^{57}$ introduces a model wherein stimulation is coupled and proportional to the pathologic synchronous rhythm. LFPs are recorded from 1 electrode and fed back into the system by an application electrode; the feedback loop contains a passive oscillator that applies a phase shift in stimulation varying according to system dynamics (e.g., latency). The passive oscillator is driven by pathologic oscillations; the phaseshifted stimulation suppresses the pathologic rhythm and vanishes as suppression is achieved. This approach has not been implemented in any clinical studies.

A related approach described previously has been used to treat ET, predicated on the observation that DBS at tremor frequency can entrain neural oscillations and modulate peripherally detected tremor amplitudes. ${ }^{10}$ This approach decouples the tremor network and was able to provide significant tremor relief with significant energy savings when compared to conventional high-frequency stimulation. ${ }^{11}$ In contrast to amplitude-responsive approaches, such modelbased approaches to feedback can, in principle, spare other neural activities that are not phase-locked to stimulation frequencies.

\section{Other Approaches}

Modulation of stimulation parameters other than amplitude via closed-loop control has not been well characterized. One potentially interesting parameter to modulate may be frequency of stimulation.

There are some data that low-frequency $(60 \mathrm{~Hz})$ stimulation may prove less likely to alter verbal fluency and gait when compared to higher-frequency $(130 \mathrm{~Hz})$ stimulation. ${ }^{17,40,60,61}$ The literature reveals variation in patient response, ${ }^{40}$ potentially transient effectiveness, ${ }^{51}$ and often decreased effectiveness in treating appendicular symptoms, tempering enthusiasm for application of low-frequency 
stimulation in current, open-loop DBS. Modulation of stimulation frequency is potentially more interesting when combined with a closed-loop approach that incorporates the ability to extract behavioral states from an implanted DBS electrode.

There is evidence that LFP signals in the STN can be used identify a variety of behaviors, including speech, motor, and random movement with up to $73.2 \%$ accuracy. ${ }^{44}$ STN LFP features, such as synchronization and interhemispheric connectivity features based on wavelet transform and Granger causality approaches, have achieved an average accuracy of $99.8 \%$ for movement identification, and $81.5 \%$ for laterality classification. ${ }^{36}$ In chronic recordings in DBS patients with akinetic rigid PD, a trend toward decreased beta power when walking was exhibited. ${ }^{50}$ The ability to use LFP features in STN to distinguish between behavioral states, if coupled to modulation of stimulation parameters such as frequency, which may be associated with a differential effect on axial versus appendicular symptoms, may provide another method for feedback control of a closed-loop DBS system that is responsive to patient needs.

\section{Discussion}

Closed-loop DBS seeks to improve an already effective therapy by coupling patient physiology to therapy delivered. To do so, an estimation of the patient's physiological state is needed, as well as control algorithms to modify and deliver therapy and assess the effects of that therapy. Differing clinical patterns may be characterized by different neural biomarkers, ${ }^{28,37,49}$ and it may be the case that patient-specific models incorporating multiple markers may be needed for the most effective clinical outcomes.

The long-term efficacy and efficiency of even the most mature attempts at closed-loop control is not established. In general, closed-loop DBS has been shown to use less power than an open system. ${ }^{34}$ However, most mature systems have thus far outsourced processing to an external computer system. ${ }^{11,23,32,34,35}$ For less-mature technologies, such as the wireless fast-scan cyclic voltammetry systems, the added power costs are completely unknown. Development of more efficient and patient-friendly rechargeable systems is likely to mitigate this concern.

Importantly, even the most mature closed-loop approaches rely on the open-loop model to determine stimulation parameters. As new DBS systems with currentsteering capabilities become increasingly popular, the search space that must be explored to determine "optimal" parameters, even in a static setting, will increase dramatically. Development of algorithms to allow automated DBS programming will be needed to fully realize the benefits of this technology.

Ultimately, the appeal of closed-loop DBS lies in the potential to treat symptoms in response to patient physiology. This may be implemented in a variety of ways, and will be facilitated by technological advances in sensor technology, in the machine-learning algorithms used to extract relevant signal features and to identify and respond to changes in estimated patient state, in the hardware used to deliver that stimulation, and by our increasing under- standing of the cortical and subcortical dynamics underlying the pathologies treated.

\section{Conclusions}

DBS is a successful therapy for movement disorders such as Parkinson's disease, essential tremor, and dystonia. Traditional DBS therapy is open loop; parameters are determined and adjusted in a clinical setting and do not, for the most part, take into account variations in patient symptoms and need for stimulation. Closed-loop DBS seeks to employ biomarkers, derived from sensors such as EMG, ECoG, and LFP, to optimize stimulation parameters for clinical treatment. Preliminary data suggest that patientresponsive stimulation may provide superior symptom relief compared with open-loop DBS, may mitigate some of the side effects of DBS, and may be more power efficient than constant stimulation. However, patient-responsive therapy for movement disorders is yet in the early stages of development, requiring additional studies to determine what approaches are feasible, efficacious, and efficient.

\section{References}

1. Air EL, Ryapolova-Webb E, de Hemptinne C, Ostrem JL, Galifianakis NB, Larson PS, et al: Acute effects of thalamic deep brain stimulation and thalamotomy on sensorimotor cortex local field potentials in essential tremor. Clin Neurophysiol 123:2232-2238, 2012

2. Albanese A, Barnes MP, Bhatia KP, Fernandez-Alvarez E, Filippini G, Gasser T, et al: A systematic review on the diagnosis and treatment of primary (idiopathic) dystonia and dystonia plus syndromes: report of an EFNS/MDS-ES Task Force. Eur J Neurol 13:433-444, 2006

3. Aronson S, Geddes LA: Electrode potential stability. IEEE Trans Biomed Eng 32:987-988, 1985

4. Bartolomei F, Lagarde S, Wendling F, McGonigal A, Jirsa V, Guye M, et al: Defining epileptogenic networks: Contribution of SEEG and signal analysis. Epilepsia 58:1131-1147, 2017

5. Basu I, Graupe D, Tuninetti D, Shukla P, Slavin KV, Metman LV, et al: Pathological tremor prediction using surface electromyogram and acceleration: potential use in 'ON-OFF' demand driven deep brain stimulator design. J Neural Eng 10:036019, 2013

6. Bédard C, Kröger H, Destexhe A: Modeling extracellular field potentials and the frequency-filtering properties of extracellular space. Biophys J 86:1829-1842, 2004

7. Benabid AL, Pollak P, Gao D, Hoffmann D, Limousin P, Gay E, et al: Chronic electrical stimulation of the ventralis intermedius nucleus of the thalamus as a treatment of movement disorders. J Neurosurg 84:203-214, 1996

8. Bronte-Stewart HM, Ding L, Alexander C, Zhou Y, Moore GP: Quantitative digitography (QDG): a sensitive measure of digital motor control in idiopathic Parkinson's disease. Mov Disord 15:36-47, 2000

9. Brown P, Williams D: Basal ganglia local field potential activity: character and functional significance in the human. Clin Neurophysiol 116:2510-2519, 2005

10. Cagnan H, Brittain JS, Little S, Foltynie T, Limousin P, Zrinzo L, et al: Phase dependent modulation of tremor amplitude in essential tremor through thalamic stimulation. Brain 136:3062-3075, 2013

11. Cagnan H, Pedrosa D, Little S, Pogosyan A, Cheeran B, Aziz T, et al: Stimulating at the right time: phase-specific deep brain stimulation. Brain 140:132-145, 2017

12. Chang SY, Kim I, Marsh MP, Jang DP, Hwang SC, Van Gompel JJ, et al: Wireless fast-scan cyclic voltammetry to monitor 
adenosine in patients with essential tremor during deep brain stimulation. Mayo Clin Proc 87:760-765, 2012

13. de Hemptinne C, Ryapolova-Webb ES, Air EL, Garcia PA, Miller KJ, Ojemann JG, et al: Exaggerated phase-amplitude coupling in the primary motor cortex in Parkinson disease. Proc Natl Acad Sci U S A 110:4780-4785, 2013

14. DeLong MR, Wichmann T: Circuits and circuit disorders of the basal ganglia. Arch Neurol 64:20-24, 2007

15. Deuschl G, Schade-Brittinger C, Krack P, Volkmann J, Schäfer H, Bötzel K, et al: A randomized trial of deep-brain stimulation for Parkinson's disease. N Engl J Med 355:896908, 2006

16. Ellis RJ, Ng YS, Zhu S, Tan DM, Anderson B, Schlaug G, et al: A Validated Smartphone-Based Assessment of Gait and Gait Variability in Parkinson's Disease. PLoS One 10:e0141694, 2015

17. Fagundes VC, Rieder CR, da Cruz AN, Beber BC, Portuguez MW: Deep brain stimulation frequency of the subthalamic nucleus affects phonemic and action fluency in Parkinson's disease. Parkinsons Dis 2016:6760243, 2016

18. Geller EB, Skarpaas TL, Gross RE, Goodman RR, Barkley GL, Bazil CW, et al: Brain-responsive neurostimulation in patients with medically intractable mesial temporal lobe epilepsy. Epilepsia 58:994-1004, 2017

19. Graupe D, Basu I, Tuninetti D, Vannemreddy P, Slavin KV: Adaptively controlling deep brain stimulation in essential tremor patient via surface electromyography. Neurol Res 32:899-904, 2010

20. Hariz GM, Limousin P, Hamberg K: "DBS means everything - for some time". Patients' perspectives on daily life with deep brain stimulation for Parkinson's disease. J Parkinsons Dis 6:335-347, 2016

21. Hariz MI, Rehncrona S, Quinn NP, Speelman JD, Wensing C: Multicenter study on deep brain stimulation in Parkinson's disease: an independent assessment of reported adverse events at 4 years. Mov Disord 23:416-421, 2008

22. Herron JA, Thompson MC, Brown T, Chizeck HJ, Ojemann JG, Ko AL: Chronic electrocorticography for sensing movement intention and closed-loop deep brain stimulation with wearable sensors in an essential tremor patient. J Neurosurg 127:580-587, 2017

23. Herron JA, Thompson MC, Brown T, Chizeck HJ, Ojemann JG, Ko AL: Cortical brain-computer interface for closed-loop deep brain stimulation. IEEE Trans Neural Syst Rehabil Eng 25:2180-2187, 2017

24. Kajikawa Y, Schroeder CE: How local is the local field potential? Neuron 72:847-858, 2011

25. Karamintziou SD, Deligiannis NG, Piallat B, Polosan M, Chabardès S, David O, et al: Dominant efficiency of nonregular patterns of subthalamic nucleus deep brain stimulation for Parkinson's disease and obsessive-compulsive disorder in a data-driven computational model. J Neural Eng 13:016013, 2016

26. Kishida KT, Sandberg SG, Lohrenz T, Comair YG, Sáez I, Phillips PE, et al: Sub-second dopamine detection in human striatum. PLoS One 6:e23291, 2011

27. Kondylis ED, Randazzo MJ, Alhourani A, Lipski WJ, Wozny TA, Pandya Y, et al: Movement-related dynamics of cortical oscillations in Parkinson's disease and essential tremor. Brain 139:2211-2223, 2016

28. Kühn AA, Tsui A, Aziz T, Ray N, Brücke C, Kupsch A, et al: Pathological synchronisation in the subthalamic nucleus of patients with Parkinson's disease relates to both bradykinesia and rigidity. Exp Neurol 215:380-387, 2009

29. Lee CY, Kang SJ, Hong SK, Ma HI, Lee U, Kim YJ: A validation study of a smartphone-based finger tapping application for quantitative assessment of bradykinesia in Parkinson's disease. PLoS One 11:e0158852, 2016

30. Lee KH, Chang SY, Jang DP, Kim I, Goerss S, Van Gompel
$\mathrm{J}$, et al: Emerging techniques for elucidating mechanism of action of deep brain stimulation. Conf Proc IEEE Eng Med Biol Soc 2011:677-680, 2011

31. Lee KH, Lujan JL, Trevathan JK, Ross EK, Bartoletta JJ, Park HO, et al: WINCS Harmoni: Closed-loop dynamic neurochemical control of therapeutic interventions. Sci Rep 7:46675, 2017

32. Little S, Beudel M, Zrinzo L, Foltynie T, Limousin P, Hariz $\mathrm{M}$, et al: Bilateral adaptive deep brain stimulation is effective in Parkinson's disease. J Neurol Neurosurg Psychiatry 87:717-721, 2016

33. Little S, Pogosyan A, Neal S, Zavala B, Zrinzo L, Hariz M, et al: Adaptive deep brain stimulation in advanced Parkinson disease. Ann Neurol 74:449-457, 2013

34. Little S, Tripoliti E, Beudel M, Pogosyan A, Cagnan H, Herz D, et al: Adaptive deep brain stimulation for Parkinson's disease demonstrates reduced speech side effects compared to conventional stimulation in the acute setting. J Neurol Neurosurg Psychiatry 87:1388-1389, 2016

35. Malekmohammadi M, Herron J, Velisar A, Blumenfeld Z, Trager MH, Chizeck HJ, et al: Kinematic adaptive deep brain stimulation for resting tremor in Parkinson's disease. Mov Disord 31:426-428, 2016

36. Mamun KA, Mace M, Lutman ME, Stein J, Liu X, Aziz T, et al: Movement decoding using neural synchronization and inter-hemispheric connectivity from deep brain local field potentials. J Neural Eng 12:056011, 2015

37. Marceglia S, Fumagalli M, Priori A: What neurophysiological recordings tell us about cognitive and behavioral functions of the human subthalamic nucleus. Expert Rev Neurother 11:139-149, 2011

38. Merrill DR, Bikson M, Jefferys JG: Electrical stimulation of excitable tissue: design of efficacious and safe protocols. $\mathbf{J}$ Neurosci Methods 141:171-198, 2005

39. Montague PR, Hyman SE, Cohen JD: Computational roles for dopamine in behavioural control. Nature 431:760-767, 2004

40. Moreau C, Defebvre L, Destée A, Bleuse S, Clement F, Blatt $\mathrm{JL}$, et al: STN-DBS frequency effects on freezing of gait in advanced Parkinson disease. Neurology 71:80-84, 2008

41. Nathan DA, Center S, Wu CY, Keller W: An implantable synchronous pacemaker for the long term correction of complete heart block. Am J Cardiol 11:362-367, 1963

42. Neumann WJ, Degen K, Schneider GH, Brücke C, Huebl J, Brown P, et al: Subthalamic synchronized oscillatory activity correlates with motor impairment in patients with Parkinson's disease. Mov Disord 31:1748-1751, 2016

43. Neumann WJ, Staub-Bartelt F, Horn A, Schanda J, Schneider $\mathrm{GH}$, Brown P, et al: Long term correlation of subthalamic beta band activity with motor impairment in patients with Parkinson's disease. Clin Neurophysiol 128:2286-2291, 2017

44. Niketeghad S, Hebb AO, Nedrud J, Hanrahan SJ, Mahoor MH: Single trial behavioral task classification using subthalamic nucleus local field potential signals. Conf Proc IEEE Eng Med Biol Soc 2014:3793-3796, 2014

45. Perge JA, Homer ML, Malik WQ, Cash S, Eskandar E, Friehs $\mathrm{G}$, et al: Intra-day signal instabilities affect decoding performance in an intracortical neural interface system. J Neural Eng 10:036004, 2013

46. Popovych OV, Lysyansky B, Rosenblum M, Pikovsky A, Tass PA: Pulsatile desynchronizing delayed feedback for closedloop deep brain stimulation. PLoS One 12:e0173363, 2017

47. Popovych OV, Lysyansky B, Tass PA: Closed-loop deep brain stimulation by pulsatile delayed feedback with increased gap between pulse phases. Sci Rep 7:1033, 2017

48. Popovych OV, Tass PA: Multisite delayed feedback for electrical brain stimulation. Front Physiol 9:46, 2018

49. Priori A, Foffani G, Pesenti A, Tamma F, Bianchi AM, Pel- 
legrini M, et al: Rhythm-specific pharmacological modulation of subthalamic activity in Parkinson's disease. Exp Neurol 189:369-379, 2004

50. Quinn EJ, Blumenfeld Z, Velisar A, Koop MM, Shreve LA, Trager MH, et al: Beta oscillations in freely moving Parkinson's subjects are attenuated during deep brain stimulation. Mov Disord 30:1750-1758, 2015

51. Ricchi V, Zibetti M, Angrisano S, Merola A, Arduino N, Artusi CA, et al: Transient effects of $80 \mathrm{~Hz}$ stimulation on gait in STN DBS treated PD patients: a 15 months follow-up study. Brain Stimul 5:388-392, 2012

52. Rowland NC, De Hemptinne C, Swann NC, Qasim S, Miocinovic S, Ostrem JL, et al: Task-related activity in sensorimotor cortex in Parkinson's disease and essential tremor: changes in beta and gamma bands. Front Hum Neurosci 9:512, 2015

53. Samà A, Pérez-Lopez C, Romagosa J, Rodríguez-Martín D, Català A, Cabestany J, et al: Dyskinesia and motor state detection in Parkinson's disease patients with a single movement sensor. Conf Proc IEEE Eng Med Biol Soc 2012:1194-1197, 2012

54. Shukla P, Basu I, Graupe D, Tuninetti D, Slavin KV: A neural network-based design of an on-off adaptive control for Deep Brain Stimulation in movement disorders. Conf Proc IEEE Eng Med Biol Soc 2012:4140-4143, 2012

55. Skoch J, Adelson PD, Bhatia S, Greiner HM, Rydenhag B, Scavarda D, et al: Subdural grid and depth electrode monitoring in pediatric patients. Epilepsia 58 (Suppl 1):56-65, 2017

56. Swann NC, de Hemptinne C, Miocinovic S, Qasim S, Wang SS, Ziman N, et al: Gamma oscillations in the hyperkinetic state detected with chronic human brain recordings in Parkinson's disease. J Neurosci 36:6445-6458, 2016

57. Tukhlina N, Rosenblum M, Pikovsky A, Kurths J: Feedback suppression of neural synchrony by vanishing stimulation.

Phys Rev E Stat Nonlin Soft Matter Phys 75:011918, 2007

58. Wichmann T, DeLong MR, Guridi J, Obeso JA: Milestones in research on the pathophysiology of Parkinson's disease. Mov Disord 26:1032-1041, 2011

59. Wingeier B, Tcheng T, Koop MM, Hill BC, Heit G, BronteStewart HM: Intra-operative STN DBS attenuates the prominent beta rhythm in the STN in Parkinson's disease. Exp Neurol 197:244-251, 2006

60. Xie T, Padmanaban M, Bloom L, MacCracken E, Bertacchi B, Dachman A, et al: Effect of low versus high frequency stimulation on freezing of gait and other axial symptoms in Parkinson patients with bilateral STN DBS: a mini-review. Transl Neurodegener 6:13, 2017

61. Xie T, Vigil J, MacCracken E, Gasparaitis A, Young J, Kang $\mathrm{W}$, et al: Low-frequency stimulation of STN-DBS reduces aspiration and freezing of gait in patients with PD. Neurology 84:415-420, 2015

62. Yamamoto T, Katayama Y, Ushiba J, Yoshino H, Obuchi T, Kobayashi K, et al: On-demand control system for deep brain stimulation for treatment of intention tremor. Neuromodulation 16:230-235, 2013

\section{Disclosures}

The authors report no conflict of interest concerning the materials or methods used in this study or the findings specified in this paper.

\section{Author Contributions}

Conception and design: Ko. Acquisition of data: Kuo. Drafting the article: Ko, Kuo, White-Dzuro. Reviewed submitted version of manuscript: Ko, Kuo. Approved the final version of the manuscript on behalf of all authors: Ko.

\section{Correspondence}

Andrew L. Ko: University of Washington, Seattle, WA. alko00@ neurosurgery.washington.edu. 